\section{A-form DNA for survival}

SIRV2 is a double-stranded DNA virus that infects Sulfolobus islandicus, an archaeon that lives in an environment with temperatures around $80^{\circ} \mathrm{C}$ and a $\mathrm{pH}$ of 3. Egelman, Prangishvili and colleagues have now investigated how SIRV2 maintains its genomic integrity under such extreme conditions. The rodshaped virion was known to be composed of thousands of copies of a small DNA-binding capsid protein. Using cryo-EM and comparative modeling (RosettaCM), the researchers reconstructed the structure of the SIRV2 virion at $\sim 4$ resolution. The structure revealed that the DNA is in A form and is encapsulated by the N-terminal portion of the capsid protein, which is unstructured in solution but folded into helix-turn-helix motifs in the virion. The helices from neighboring protein subunits are packed in an antiparallel, interdigitated arrangement, and they wrap tightly around the DNA, interacting with the phosphate backbone via conserved polar and hydrophobic residues. Along with hydrophobic interprotein interactions, this arrangement keeps the DNA completely surrounded by protein and inaccessible to solvent. These features are reminiscent of early observations on the Bacillus subtilis spore, a dormant form of the bacterium that shows high resistance to heat, radiation, chemical treatments and desiccation. Within B. subtilis spores, the genomic DNA is in complex with small acid-soluble proteins (SASPs), which can induce a transition of double-stranded DNA from B to A form in vitro. SASPs and SIRV2 protein share no detectable sequence or structural homology, and the functional convergence indicates that adopting A-form DNA might be a widespread biological mechanism allowing organisms to withstand adverse conditions. (Science 348, 914-917, 2015)

\section{An unexpected role for mitochondrial ClpX}

The AAA+ unfoldase ClpX contributes to bacterial proteostasis. In eukaryotes ClpX is found only in mitochondria (mtClpX), and its function has so far remained elusive. Baker and colleagues now report that $\mathrm{mtClpX}$ plays a part in heme biosynthesis by facilitating synthesis of the precursor 5-aminolevulinic acid (ALA). Searching large-scale genetic- and chemical-interaction data in Saccharomyces cerevisiae, they found that the interaction profile of the gene encoding the yeast mtClpX homolog Mcx1 is strongly correlated with those of the genes involved in the initial steps of heme biosynthesis, including HEM1, which encodes the ALA synthase Hem1. Deletion or functionimpairing mutation of Mcxl reduces cellular heme levels, but the phenotype is rescued by supplementation with ALA, thus suggesting a direct role of Mcx1 in ALA synthesis. ALA synthases, including Hem1, require the cofactor pyridoxal phosphate (PLP) for enzymatic activity. Baker and colleagues found that Mcx 1 directly interacts with Hem 1 and increases PLP binding to the apo-Hem 1 by eightfold, thus facilitating its activation. Although PLP binding requires Hem 1 to maintain at least a partially folded state, Hem 1 activation is nevertheless dependent on the unfoldase function of Mcx 1. These observations led the authors to propose that upon interacting with the apoenzyme, mtClpX may locally unfold ALA synthase, thus exposing its buried active site to facilitate PLP binding. Consistently with the high sequence conservation of $\mathrm{mtClpX}$ and ALA synthase in yeast and mammals, mouse mtClpX also stimulates PLP activation of a human ALA synthase apoenzyme in vitro; this suggests that the mechanism identified in yeast is conserved across eukaryotes. Finally, Baker and colleagues found that knockdown of mtClpX in zebrafish embryos impairs erythropoiesis, consistently with the high requirement for heme to form hemoglobin during this process. These findings shed light on the role of ClpX in mitochondria and indicate an unexpected function for AAA+ unfoldases. (Cell 161, 858-867, 2015) $C D$

\section{The ghost in the machine}

The human genome bears traces of past retroviral infections in the form of endogenous retroviruses (ERVs) whose long terminal repeats (LTRs) contain cis-regulatory elements that affect gene activity. The evolutionarily most recent member of the human ERV family, HERVK, retains intact open reading frames (ORFs) encoding retroviral proteins that are normally silenced by DNA methylation. Now Wysocka and colleagues show that HERVK transcription is activated during human embryogenesis, with likely effects on early development. Analysis of single-cell RNA-sequencing profiles at different embryonic stages revealed active HERVK transcription at the eight-cell stage and in epiblast cells (ECs) of the preimplantation blastocyst; this transcription is then silenced upon blastocyst outgrowth. Similarly, HERVK transcripts are present in naive human embryonic stem cells (ESCs) that correspond to the preimplantation state but not in primed ESCs, thus suggesting that HERVK expression is induced during a transient window when DNA is hypomethylated and regulatory elements are accessible to the pluripotency factors OCT4 and SOX2. Indeed, the authors show that the LTR of HERVK contains an OCT4-binding motif that is engaged by the transcription factor in ECs but not primed ESCs. DNA hypomethylation is required for OCT4-dependent activation, which promotes stage-specific expression of proviral proteins. Most remarkably, transmission electron microscopy shows that human blastocysts contain numerous viral-like particles. Moreover, expression of HERVK Rec, a protein that exports viral RNAs to the cytosol for translation, induces innateantiviral-response components in ECs and increases the levels of the interferon-induced viral restriction factor IFITM1. Importantly, Rec overexpression is sufficient to confer resistance to influenza virus infection in EC cell lines, thus suggesting that HERVK might provide an immunoprotective effect during preimplantation development. Wysocka and colleagues also identified 1,600 cellular RNAs that are bound by Rec, and ribosome profiling suggested that their translation is stimulated by Rec association. The finding that HERVK proteins are expressed in early embryogenesis and are able to influence host factors reveals an unexpected role for products of ancient retroviral infection during human embryonic development. (Nature doi:10.1038/nature14308, 20 April 2015) 